\title{
Four Year Effects of Couple Relationship Education on Low and High Satisfaction Couples: A Randomized Clinical Trial
}

\author{
W. Kim Halford \\ University of Queensland \\ Riyad H. Rahimullah \\ University of Queensland \\ Keithia L. Wilson \\ Griffith University \\ Stefano Occhipinti \\ Griffith University \\ Dean M. Busby

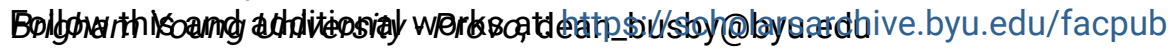 \\ Part of the Other Social and Behavioral Sciences Commons
}

See next page for additional authors

Original Publication Citation

Halford, W. K., Rahimullah, R., Wilson, K. L., Occhipinti, S., Busby, D. M., \& Larson, J. (2017). Four year effects of couple relationship education on low and high satisfaction couples: A randomized clinical trial. Journal of Consulting and Clinical Psychology, 85, 495-507.

\section{BYU ScholarsArchive Citation}

Halford, W. Kim; Rahimullah, Riyad H.; Wilson, Keithia L.; Occhipinti, Stefano; Busby, Dean M.; and Larson, Jeffrey, "Four Year Effects of Couple Relationship Education on Low and High Satisfaction Couples: A Randomized Clinical Trial" (2017). Faculty Publications. 4630.

https://scholarsarchive.byu.edu/facpub/4630

This Peer-Reviewed Article is brought to you for free and open access by BYU ScholarsArchive. It has been accepted for inclusion in Faculty Publications by an authorized administrator of BYU ScholarsArchive. For more information, please contact ellen_amatangelo@byu.edu. 


\section{Authors}

W. Kim Halford, Riyad H. Rahimullah, Keithia L. Wilson, Stefano Occhipinti, Dean M. Busby, and Jeffrey Larson

This peer-reviewed article is available at BYU ScholarsArchive: https://scholarsarchive.byu.edu/facpub/4630 


\title{
Four Year Effects of Couple Relationship Education on Low and High Satisfaction Couples: A Randomized Clinical Trial
}

\author{
W. Kim Halford and Riyad H. Rahimullah \\ University of Queensland
}

\author{
Keithia L. Wilson and Stefano Occhipinti \\ Griffith University
}

\author{
Dean M. Busby and Jeffry Larson \\ Brigham Young University
}

\begin{abstract}
Objective: Relationship education (RE) usually is conceived of as relationship enhancement for currently satisfied couples, with a goal of helping couples sustain satisfaction. However, RE also might be useful as a brief, indicated intervention for couples with low satisfaction. The current study evaluated the effect of RE on couples with low and high relationship satisfaction. Method: The study was a randomized controlled trial in which 182 couples were randomly assigned to: a book reading control condition (control); RELATE online assessment with feedback and relationship goal setting (RELATE); or RELATE with CoupleCARE (RCC), a flexible delivery skill-based education program. Couples were assessed on relationship satisfaction and individual mental health before and after RE, and through to 4-year follow-up. Results: Couples with high initial satisfaction showed no effects of RE on satisfaction. RCC but not RELATE increased satisfaction in couples with low initial satisfaction, but effects dissipated between 6 and 12 months after RE. There were no effects of RE on mental health. Conclusion: Flexible delivery RE produces immediate effects as an indicated early intervention for couples with low relationship satisfaction, but the effects attenuate. Future research needs to seek methods to produce better maintained effects.
\end{abstract}

What is the public health significance of this article?

Flexible delivery relationship education (RE) has no demonstrable benefit for couples high in satisfaction, and universal offering is not justified at this time. RE enhances relationship satisfaction in couples with low relationship satisfaction, but that effect dissipates across 6 to 12 months. Indicated offering of RE might be warranted, but there needs to be further research on how best to enhance maintenance of effects.

Keywords: relationship education, relationship distress, couple relationship

Couple relationship education (RE) usually has been offered universally, and conceived of as relationship enhancement for currently satisfied couples (Halford \& Bodenmann, 2013). However, RE also has short-term benefits as an indicated intervention for couples with low satisfaction (Halford et al., 2015). The current article is a randomized controlled trial that tested whether couples with low relationship satisfaction show meaningful long-term gains in relationship satisfaction or individual mental health after RE.

\section{Couple RE}

RE was developed to enrich couples' relationships and help them sustain a mutually satisfying and stable relationship (Halford, Markman, \& Stanley, 2008). There are two general approaches to RE that are evidence-based and widely adopted: assessment with feedback and curriculum-based education (Halford, 2011). Assessment with feedback is based on the assumption that providing couples with key understandings about their relationship will en-
This article was published Online First March 13, 2017.

W. Kim Halford and Riyad H. Rahimullah, School of Psychology, University of Queensland; Keithia L. Wilson and Stefano Occhipinti, School of Psychology, Griffith University; Dean M. Busby and Jeffry Larson, School of Family Life, Brigham Young University.

The trial reported in this article was not registered before being conducted. We thank Ruth Bouma, Charles Farrugia, Philippa Garrity, and Chris Pepping for assistance with the conduct of the study. The conduct of research reported in this article was supported by an Australian Research Council Discovery Project Grant 1095507, The Effects of Home-Based Couple Relationship Education, to W. Kim Halford and Keithia L. Wilson.

W. Kim Halford and Keithia L. Wilson are both authors of the CoupleCARE program and receive royalties from the sale of the program. Jeff Larson is the author of The Great Marriage Tune Up, and receive royalties from the sale of that book. Jeff Larson and Dean Busby are coauthors of RELATE and receive royalties from the RELATE Institute for the use of the program.

Correspondence concerning this article should be addressed to W. Kim Halford, School of Psychology, University of Queensland, Qld 4072, Australia. E-mail: K.Halford@psy.uq.edu.au 
able them to make any required changes to strengthen their relationship. Curriculum-based education is based on the assumption that teaching couples key relationship skills and knowledge will empower them to sustain a satisfying relationship.

Evidence-based RE usually is brief. Assessment with feedback can involve as little as 2 hours of client contact but more often is 4 to 6 hours (Halford, 2011). Curriculum-based RE typically involves 12 to 15 hours that introduce key relationship knowledge (e.g., developing shared, realistic relationship expectations) and skills (e.g., couple communication; Halford et al., 2008). RE typically has a relatively fixed curriculum (Markman \& Ritchie, 2015), although there is some tailoring of content to individual couple needs. For example, in the CoupleCARE program (Halford, 2016) partners select self-change goals to work on as part of the program.

$\mathrm{RE}$ is somewhat distinct from couple therapy. The latter is usually addressed to couples with relationship distress. In contrast to the relatively fixed curriculum of RE, evidence-based couple therapy typically involves developing a couple-specific conceptualization of distress, providing tailored treatment, and the treatment is often extensive in duration, with evidence-based approaches often involving 20 or more sessions of therapy (Snyder \& Halford, 2012).

Although RE is somewhat distinct from couple therapy, there are two ways in which there is considerable overlap. First, the content typical of curriculum-based RE and couple therapy is quite similar. For example, cognitive-behavioral couple therapy includes a focus on enhancing shared enjoyable activities, teaching couple communication, changing unhelpful couple interaction cycles, and identifying and challenging unhelpful cognitions (Snyder \& Halford, 2012). The content of evidence-based RE programs like the Positive Relationship Education Program (PREP), CoupleCARE, and Couple Coping Enhancement Training include many of these same content areas (Halford \& Bodenmann, 2013).

A second way in which RE and couple therapy overlap is that, among couples attending RE, there is a substantial proportion of couples with low relationship satisfaction, with some couples meeting criteria for clinical distress (DeMaria, 2005). The proportion of low satisfaction couples depends upon the outreach strategies used to attract couples to RE. Most often RE has been offered as a universal intervention to all couples in a committed relationship (Halford \& Bodenmann, 2013). For example, RE is widely available in the United States to couples who are soon to marry (Stanley, Amato, Johnson, \& Markman, 2006). In such universal offerings, the vast majority of couples attending have high relationship satisfaction, and there is some evidence that couples most at risk for future deterioration of relationship satisfaction are underrepresented (Halford, O’Donnell, Lizzio, \& Wilson, 2006: Doss, Rhoades, Stanley, Markman, \& Johnson, 2009a). Sometimes RE has been offered selectively to high-risk couples; these couples often have high satisfaction at the time of presentation but are assessed as being of high risk for later deterioration in satisfaction. For example, Petch, Halford, Creedy, and Gamble (2012) offered RE to couples having their first child when many couples experience declining relationship satisfaction, and they assessed RE effects on couples who had further risk factors, such as an unplanned pregnancy or a history of depression in either partner (Petch et al., 2012). Finally, RE can be offered as an indicated intervention with couples who already have somewhat low satisfaction (e.g., Cordova et al., 2014).

The potential effects of RE as an indicated intervention for low satisfaction couples are potentially important. In many developed countries, (e.g., the United States, Japan, Australia, and Norway), government and community agencies disseminate RE in an attempt to reduce the negative effects of relationship distress (Markman \& Ritchie, 2015). The rates of attendance of RE have grown markedly; in the United States only about $12 \%$ of marrying couples received RE in the 1950s versus $44 \%$ in the 1990s (Stanley et al., 2006). Furthermore, RE attracts substantial numbers of couples with relationship distress (DeMaria, 2005), many of whom express reservations about seeking couple therapy (Morrill et al., 2011).

In addition to the potential positive effects of RE on low relationship satisfaction, RE might benefit mental health. There is a strong association between being in a mutually satisfying couple relationship and positive mental health in the partners (Whisman, 2013), and RE that enhances relationship satisfaction might also enhance mental health (Halford et al., 2008).

A caveat on the potential benefit of RE for couples with low relationship satisfaction is that relationship distress seems to be taxonomic (Whisman, Beach, \& Snyder, 2008). That is, there seems to be an underlying categorical difference between distressed and satisfied couples. Some authors suggest that RE might not be effective in assisting distressed couples, and that special screening and procedures are needed if RE is attracting distressed couples (e.g., Bradford, Hawkins, \& Acker, 2015). However, one advantage of existing forms of RE is that they are promoted as being for normal healthy couples, which is believed to reduce stigma in attending (Markman \& Ritchie, 2015). Before undertaking expensive changes to RE that might make it seem more clinical in approach, it is important to test whether existing RE improves satisfaction in couples with low relationship satisfaction.

\section{Effects of Couple Relationship Education}

Assessment with feedback. A quasi-experimental evaluation (Knutson \& Olson, 2003), and one randomized controlled trial showed immediate increases in relationship satisfaction from an inventory-based assessment with feedback (Larson et al., 2007). Extending to more multimodal assessment as the basis for feedback, Worthington and colleagues (1995) had couples complete self-report inventories, a communication task, and a selfmonitoring diary across three sessions, and they found assessment, feedback, and goal setting produced immediate increases in relationship satisfaction. Building upon these earlier studies, Cordova and colleagues found the Relationship Check-up, which is four sessions consisting of assessment with feedback plus brief skillfocused interventions, produced immediate increases in relationship satisfaction in two randomized controlled trials (Cordova et al., 2005; Cordova et al., 2014), which was sustained for 12 months in the most recent study (Cordova et al., 2014).

Curriculum-based RE. There are mixed findings on the effects of curriculum-based RE on relationship satisfaction, ranging from medium to large effects (e.g., Halford et al., 2010; Schulz, Cowan, \& Cowan, 2006), through to null effects (e.g., Rogge, Cobb, Lawrence, Johnson, \& Bradbury, 2013; Trillingsgaard, Baucom, Heyman, \& Elklit, 2012). A meta-analysis of 117 studies of curriculum-based RE reported medium effect size improve- 
ments in couple communication, $d=.44$, and small increases in relationship satisfaction, $d=.36$, immediately after RE (Hawkins, Blanchard, Baldwin, \& Fawcett, 2008). Programs with moderate dosage (9-20 hr) had substantially larger effect sizes than low dose programs (1-8 hr). However, a more recent meta-analysis of 38 studies of the effects of RE with low income couples found only a very small mean effect on relationship satisfaction, $d=.067$, with no evidence of a reliable effect of RE on relationship stability, $d=-.002$ (Hawkins \& Erickson, 2015).

The small mean effects of RE on relationship satisfaction have been variously interpreted by reviewers as: current approaches to RE are relatively ineffective overall (Johnson \& Bradbury, 2015); that RE is ineffective with particular groups, notably low income and minority couples (Johnson, 2012); or that small sizes might well reflect that some couples (e.g., those high in satisfaction at presentation) show little improvement while other couples (e.g., those low in satisfaction at presentation) show large improvement (Halford \& Bodenmann, 2013). Consistent with this last interpretation, in the Hawkins and colleagues' (2008) meta-analysis, the effect sizes of RE were larger for samples with higher proportions of low satisfaction couples. Moreover, two recent studies found RE produced moderate to large short-term improvements in couples who had low relationship satisfaction before RE but produced little change in couples with high satisfaction before RE (Halford et al., 2015; Quirk, Strokoff, Owen, France, \& Bergen, 2014). However, the maintenance of these effects was not assessed in either study.

Halford and Bodenmann (2013) reviewed 17 randomized controlled trials of RE with follow-up of 12 months or more, and found 14 of the 17 studies reported positive effects of RE on relationship satisfaction. However, the benefits of RE seemed to be predominantly with couples who were initially somewhat low in satisfaction. For example, two long-term evaluations of RE in Germany found couples with somewhat low mean satisfaction initially showed immediate gains in satisfaction, and long-term maintenance of those gains (Braukhaus, Hahlweg, Kroeger, Groth, \& Fehm-Wolfsdorf, 2003; Kaiser, Hahlweg, Fehm-Wolfsdorf, \& Groth, 1998). However, there was considerable variability in initial satisfaction within the samples, and it is not clear if the couples with the lower satisfaction were those making the most gain. A major limitation of the research reviewed was that none examined effects on low satisfaction couples specifically, so the long-term effects of RE for low satisfaction couples is unclear.

\section{Importance of Effects of Couple RE on Low Satisfaction}

There is a small but growing literature on couple help-seeking that suggests RE has a potentially important role in assisting low satisfaction couples. While evidence-based couple therapy has a well replicated efficacy in reducing relationship distress (Snyder \& Halford, 2012), the reach of couple therapy is modest. Only about 1 in 5 divorced couples attend couple therapy before separating (Johnson et al., 2002). Moreover the couples who do present for couple therapy have experienced long standing relationship distress, which predicts drop out early from couple therapy (Mondor et al., 2013), and poor outcome even in couples who complete therapy (Owen, Duncan, Anker, \& Sparks, 2012). In other words, couple therapy often comes too late. It has been suggested that there is a need for brief intervention that couples are likely to access early before chronic severe distress has developed (Georgia $\&$ Doss, 2013). Across the first five years of marriage many more couples seek relationship help by attending RE workshops, or reading self-help books, than present for couple therapy (Doss, Rhoades, Stanley, \& Markman, 2009b). People in committed relationships report a greater willingness to attend RE rather than attend couple therapy (Duncan, Childs, \& Larson, 2010; Georgia \& Doss, 2013). RE might well be an accessible form of early intervention for couples with low relationship satisfaction.

\section{Flexible Delivery of RE}

Both assessment with feedback and curriculum-based RE are delivered predominantly face-to-face, and in the case of curriculumbased RE often involve substantial time commitment from couples for attendance plus travel (Halford, 2011). Despite increasing rates of attendance still only a minority of marrying couples attend such programs (Stanley et al., 2006). Many adults prefer to access psychological education through self-directed programs, that can be undertaken at times and places that suit participants, rather than through face-to-face programs (Lustria et al., 2013). Across diverse applications of adult education, programs structured to allow ease of access are termed "flexible delivery" programs, and often involve programs that can be completed at home, or online.

The RELATE assessment with feedback is available online, which makes it easily accessible (Larson et al., 2007). Similarly CoupleCARE is a flexible delivery curriculum-based RE program, which couples complete at home (Halford, 2011). As both these programs have evidence of their short-term efficacy (Halford et al., 2010; Larson et al., 2007), we evaluated the effects of these RE programs in the current research.

\section{Aims of the Study}

In summary, RE has content that seems appropriate to assist low satisfaction couples, RE seems to be more acceptable to many couples than seeking couple therapy, and might encourage couples to present before they have long standing relationship distress. Flexible delivery of RE has the potential to enhance reach even further. However, the long-term effects of RE on low satisfaction couples have not been assessed. In the current paper we tested the hypothesis that couples with low relationship satisfaction before RE would show sustained increases across 4 years in relationship satisfaction after assessment with feedback RE (Hypothesis 1), or curriculum-based RE (Hypothesis 2). Based on prior suggestions of little effect of RE on satisfaction with highly satisfied couples, we predicted couples with high satisfaction before RE would show no sustained increase in satisfaction after either assessment with feedback or curriculum-based RE (Hypothesis 3). Based on the observed association of relationship satisfaction with mental health, we also predicted that both forms of RE would enhance mental health (Hypothesis 4). It was unclear if initial relationship satisfaction might moderate benefits of RE for mental health, so we made no prediction but did test for a moderated effect of RE on mental health. A published paper (Halford et al., 2015) reported on the immediate effects of RE on relationship satisfaction in the trial reported in the current article. The current article reports on a 4-year follow-up evaluating long-term effects of RE on both relationship satisfaction and mental health. 


\section{Method}

\section{Participants}

Participants were 182 heterosexual couples recruited between March 2010 and July 2011 for a study evaluating couple RE delivered in couples' homes. Recruitment was through social media (Google and Facebook) and newspaper advertisements. Inclusion criteria were (a) couples were in a committed relationship (married, or cohabiting for a minimum of 6 months); (b) both partners consented to participate in the study; and (c) neither partner was attending couple therapy or reported severe interpartner violence.

Figure 1 presents the flow of participants through the study. As shown, 182 couples were randomly allocated to one of three conditions: a self-directed reading control group; RELATE assessment and feedback; or RELATE assessment with feedback plus the six unit CoupleCARE program (RCC). Of the couples allocated to RCC, seven couples withdrew prior to participating in the RELATE assessment feedback session. Two couples did not complete a RELATE assessment due to technical difficulties in accessing the online assessment, and these couples received CoupleCARE without completing the online assessment.

Measures. RELATE is a 271-item self-report assessment of couple relationship strengths and challenges that is accessed over the Internet (Busby, Holman, \& Taniguchi, 2001). RELATE scales correlate with relationship satisfaction, and predict the trajectory of relationship satisfaction in the early years of marriage (Larson et al., 2007). RELATE is completed by each partner independently and assesses demographic factors, relationship values, family of-

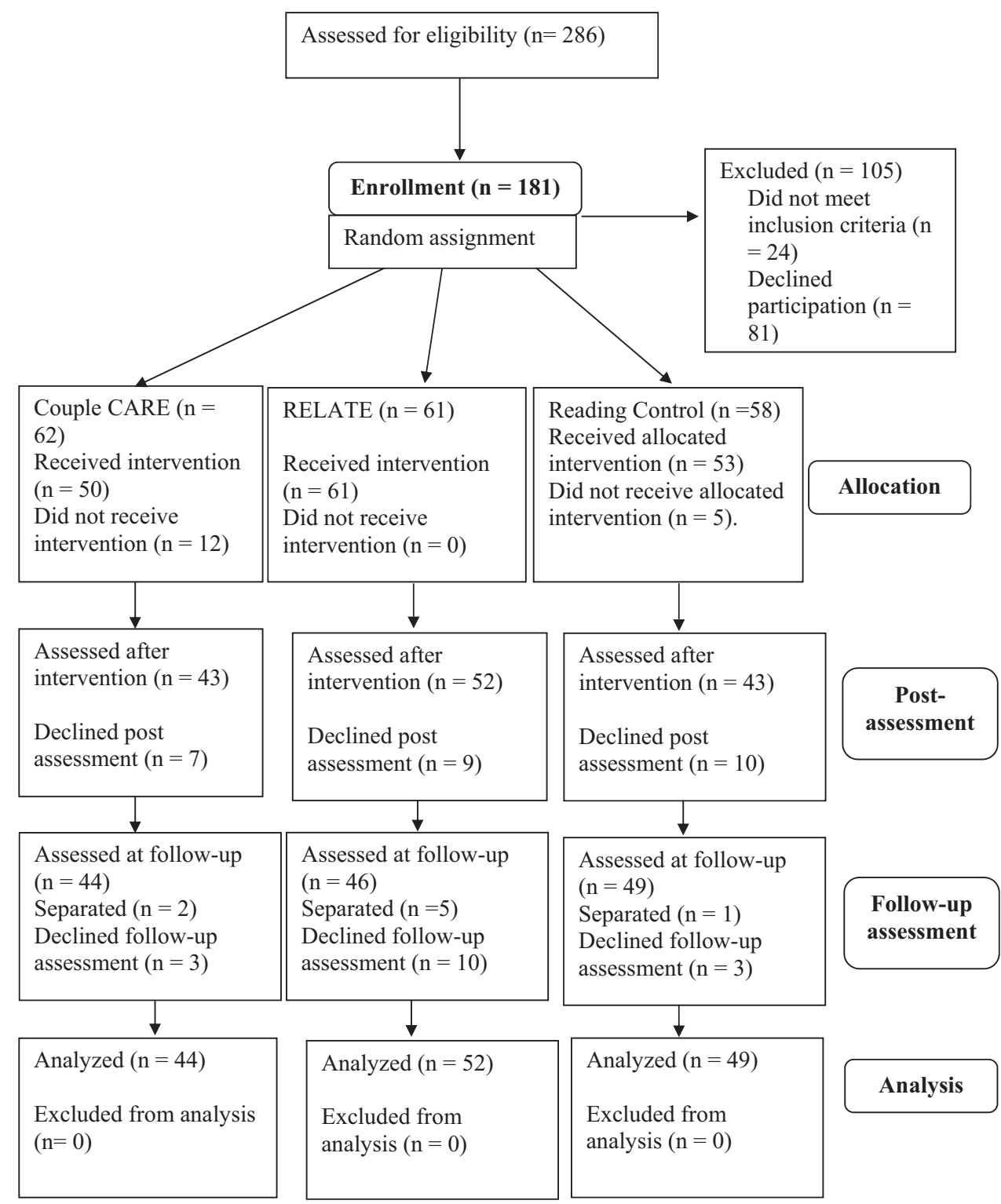

Figure 1. CONSORT flowchart of participants. 
origin experiences, and the current relationship. The RELATE system generates a report for a couple of their self-reported relationship functioning. It is used, as it was in the current study, to encourage reflection on couple-specific relationship strengths and challenges and help couples develop relationship enhancement goals (Halford, 2011).

The RELATE relationship satisfaction scale is a six-item measure of global relationship satisfaction. Each item is rated on a 5-point scale ranging from 1 (very dissatisfied) to 5 (very satisfied), high scores reflecting high satisfaction. The scale is sensitive to change resulting from RE (Halford et al., 2010). Test-retest reliability of the scale is high, $r=.78$, across a 3-week period and shows high convergent validity with other relationship satisfaction scales (Busby et al., 2001).

The Mental Health Scale (Keyes et al., 2008) is a 15-item measure of positive mental health. Each item is rated on a 6-point scale ranging from 0 (never) to 5 (every day) of how often respondents experience positive mental health indicators in the past month. The item mean score is an index of mental health. The scale has high internal reliability, moderate test-retest reliability, and convergent validity with related constructs like experience of positive affect (Keyes et al., 2008).

\section{Couple RE Programs}

Control. Couples were sent a copy of The Great Marriage Tune-Up (Larson, 2003) that they were instructed to read over a period of 6-8 weeks. The book describes the relationship influences assessed in the RELATE inventory. After approximately 3 weeks, couples received one telephone call to review their reading of the book, and encourage them to complete the reading. This condition was intended to provide couples with information without the individualized feedback with goal setting provided in RELATE, or the additional skill training provided in CoupleCARE.

RELATE assessment with feedback. Couples were sent a 13-page RELATE report. The report describes the meaning of each scale; provides a graph showing each partner's scores; and defines these scores as strengths, as neutral, or as challenges for the relationship. The final page of the report shows the scale scores on a summary graph providing an overall couple relationship profile. The procedure used was similar to that described by Larson and colleagues (2007) and was developed with the authors of RELATE. It was suggested that each partner read through the report and then discuss it together. A relationship educator then rang the couple and spoke to them in a semistructured conjoint interview about the report (see Halford, 2011 for more detail). Interviews were of 45 to 60 min duration. Partners were each asked about their overall reactions to the report, and what they identified as relationship strengths and challenges. The couple then defined relationship enhancement goals. Specifically, each partner identified two behaviors they wished to implement to enhance their relationship (e.g., "to arrange a date together" and "to ask more questions and not interrupt when discussing difficult issues").

RCC. The details of CoupleCARE delivery are described in Halford (2011). In brief, it is a six-unit program in which couples complete about one unit per week. The six units of CoupleCARE cover: relationship self-change, communication, intimacy and caring, managing differences, sexuality, and managing life changes. In RCC the telephone call at the end of the RELATE assessment explored the association between the couple's identified goals and the content of CoupleCARE. For example, the educator pointed out to couples who reported communication difficulties that Unit 2 of CoupleCARE addresses this issue. While couples completed all six units from CoupleCARE, the emphasis placed on each unit was tailored to address the specific needs of the couple.

The six units of CoupleCARE each involve the couple watching a 12- to 15-min segment of a DVD that introduces key ideas and models skills. Then the couple does a series of exercises, which are described in a guidebook, which help the couple apply the ideas and practice the skills. These tasks take approximately 45 min per unit to complete. Finally, the couple participates in a telephone call with a relationship educator who reviews their completion of the key tasks, and provides coaching and support as required. The total time commitment for couples is about $2 \mathrm{hr}$ per unit, or $12 \mathrm{hr}$ across the whole program.

Educators. The relationship educators were three qualified psychologists with extensive experience in RE delivery, and 25 postgraduate clinical psychology students at either the University of Queensland or Griffith University in Brisbane, Australia. The postgraduate clinical student educators received credit toward their course requirements of completing hours of supervised psychology practice. The educators were seven men $(22 \%)$ and 25 women (78\%). Educators completed a full day training workshop on delivery of RELATE-based feedback and CoupleCARE and had fortnightly group supervision reviewing their delivery of RE.

\section{Procedure}

Each educator was allocated blocks of three couples and these couples were, after assessment, randomly assigned to one of the three conditions. Random assignment was done by a research assistant based on a random number table. The number of blocks of couples facilitated by a single educator ranged from one to four. Conduct of the study was approved by the Human Research Ethics Committee of the University of Queensland. Participants were assessed with an online questionnaire before RE, after RE, and at 6-, 12-, 18-, 30-, and 48-months follow-up.

\section{Data Analysis}

Taking the mean of the male and female partner as an index of relationship satisfaction, couples before RE had a mean RELATE satisfaction of $22.30(S D=4.36)$. Given the previously mentioned possibility that relationship distress is taxonomic (Whisman et al., 2008), we classified couples as low or high in initial satisfaction. Low initial satisfaction was operationalized as couple satisfaction before RE that was $0.5 S D$ below the population mean $(M=23.37$, $S D=4.21)$ of a large normative sample of $n=1,056$ community couples, which was $\leq 21$ (Halford et al., 2010). We picked this cutoff as it is midway between the population mean and the $1 S D$ below that mean often used to operationalize relationship distress (Funk \& Rogge, 2007), and seemed consistent with defining the couple as of low satisfaction while encouraging indicated early intervention. Across the whole sample, $38 \%$ of couples were in that low satisfaction range, with $29 \%$ of couples being distressed (more than $1 S D$ below the population mean), satisfaction $\leq 19$.

Stata (Rabe-Hesketh \& Skrondal, 2012) was used to conduct two multilevel model analyses of intervention effects on relation- 
ship satisfaction and mental health. In both models repeated measures across time formed Level 1, partners formed Level 2, and couples formed Level 3. Time was parameterized in a piecewise manner such that in one time variable (t1) pre-RE $=0$ and all other times of assessment $=1$, and in the other time variable (t2) pre-RE and post-RE $=0$, and all other times of assessment were expressed as years since post-RE (i.e., 6-month follow-up $=0.5$ years 12 -month follow-up $=1.0$ years, etc.). These two time parameters provide an estimate of the change in scale points of the outcome variable from pre-RE to post-RE, and the slope of the trajectory of change in scale points per year from post-RE to 4-year follow-up, respectively. The equation testing the effects of RE was as follows.

$$
\begin{aligned}
& \text { Outcome }_{i j t}=\left[\beta_{0 i j}+\beta_{i j t 1}+\beta_{i j t 2}\right]+\left[\operatorname{RELATE}_{0 i j}+\operatorname{RELATE}_{i j t 1}\right. \\
& \left.+\mathrm{RELATE}_{i j t 2}+\mathrm{RCC}_{0 i j}+\mathrm{RCC}_{i j t 1}+\mathrm{RCC}_{i j t 2}\right] \\
& +\left[\text { low }- \text { satisfaction }_{0 i j}+\text { low }- \text { satisfaction }_{i j t 1}\right. \\
& \left.+ \text { low }- \text { satisfaction }_{i j t 2}\right]+[\text { RELATE } \cdot \text { low } \\
& \text { - } \text { satisfaction }_{0 i j}+\text { RELATE } \cdot \text { low }- \text { satisfaction }_{i j t 1} \\
& + \text { RELATE } \cdot \text { low }- \text { satisfaction }_{i j t 2}+\mathrm{RCC} \cdot \text { low } \\
& - \text { satisfaction }_{0 i j}+\text { RCC low }- \text { satisfaction }_{i j t 1} \\
& \left.+ \text { RCC low }-\operatorname{satisfaction}_{i j t 2}\right]
\end{aligned}
$$

Outcome $_{i j t}$ refers to either relationship satisfaction or mental health for couple $i$, partner $j$, at time t. The variables in the first set of square brackets are the unconditional growth model, $\beta_{0 i j t}$ is the intercept (the mean at pre-RE), $\beta_{i j t 1}$ is the change from pre-RE to post-RE, $\beta_{i j t 2}$ is the mean trajectory of outcome from post-RE to 4-year follow-up in scale points per year. The second set of square brackets are the effects of condition comparing the control with RELATE and then comparing the control with RCC. The third set of square brackets are the effects of low initial satisfaction $(1=$ low satisfaction, $0=$ high initial satisfaction), and the final bracket variables are the effects of the interaction of condition by low initial satisfaction. ${ }^{1}$ Fixed and random effects of intercept and time were estimated in the model.

\section{Results}

Table 1 presents the demographics of the sample by condition and pre-RE satisfaction (low or high). To test the comparability of the sample across conditions and initial satisfaction we conducted 3 two-way analyses of variance of condition (control, Relate, $\mathrm{RCC}$ ) by initial satisfaction (low or high) on male age, female age, and household annual income. There was no difference by condition on mean male age, $F(2,170)=0.429, p=.652$, female age, $F(2,170)=0.781, p=.459$, or annual income, $F(2,170)=0.804$, $p=.449$. There was no significant difference in the mean age of men between low satisfaction couples and high satisfaction couples, $F(2,170)=2.615, p=.108$. Mean age of women in low satisfaction couples $(M=43.56, S D=10.71)$ was older than women in high satisfaction couples $(M=39.60, S D=10.07), F(1$, $170)=5.589, p=.019$. Low satisfaction couples had a lower mean household income in AUD $\$ 000(M=132.25, S D=54.57)$ than high satisfaction couples $(M=157.33, S D=65.91), F(1$, $170)=6.855, p=.010$. None of the two-way interactions between condition and low-high satisfaction were significant; $F(2,170)=$ $1.299, p=.275$ for male age; $F(2,170)=1.127, p=.326$ for female age; and $F(2,170)=2.303, p=.103$ for household income.

A series of three-way $\chi^{2}$ were conducted of condition (control, Relate, RCC) by pre-RE satisfaction (low or high) on: marital status (married/cohabiting), male partner divorce (been divorced, not been divorced), female partner divorce (been divorced, not been divorced), male partner university degree completion (yes or no), female partner university degree completion (yes or no). There was no association of condition or initial satisfaction with couple marital status (married or cohabiting), $\chi^{2}(2)=1.51 p=$ .470 ; whether the male partner had been divorced, $\chi^{2}(2)=0.62$ $p=.735$; the male partner having a university degree, $\chi^{2}(2)=$ $0.21 p=.900$; or the female partner having a university degree, $\chi^{2}(2)=3.96 p=.138$. Despite random assignment there was an association of condition with whether the female partner had been divorced, $\chi^{2}(2)=6.29 p=.043$, with more women in the control having been divorced than in the other conditions. There was no association between initial satisfaction and male partner's prior divorce, $\chi^{2}(1)=0.90 p=.224$.

Overall the sample can be characterized as being aged in their early to midforties. The mean pretax annual income was AUD $\$ 150,000$, (approximately US $\$ 140,000$ ), which is about $0.5 S D$ higher than the mean income of Australian couple households with children of AUD \$127,000 (Australian Bureau of Statistics, 2011). Table 2 shows the raw means for couple relationship satisfaction, which before RE was similar to normative data for a large Australian sample $(M=23.37, S D=4.21$ ) (Halford et al., 2010). Mental health scores were similar to normative data (Keyes et al., 2008). Two-thirds of the couples were married and the rest cohabitated. The sample was more highly educated than the Australian population.

\section{Effects of RE}

The unconditional growth model on relationship satisfaction showed a fixed effect of time, $\chi^{2}(2)=35.32 p<.001$, meaning there was an overall change in mean satisfaction across time; and also a random effect of time, $\chi^{2}(2)=40.96 p<.001$, reflecting that there was variability in the extent of change across time between couples. Table 2 presents the results of the multilevel modeling comparing the three conditions, and then the moderation of effects by initial satisfaction, for relationship satisfaction and mental health. Before adding the moderation effect, there is no change in relationship satisfaction in the control or RELATE conditions, but there is a significant small to medium effect size increase in relationship satisfaction in $\mathrm{RCC}$ relative to control, $d=$ $0.45,95 \%$ confidence interval [CI: $0.18-0.69$ ], based on using the $S D=4.21$ from a large community sample (Halford et al., 2010). The trajectory of satisfaction after RE across the next 4 years corresponds to an increase of $M=2.58$ points, which is a medium effect size, $d=0.61,95 \%$ CI [0.25-0.97]. The trajectory of RELATE does not differ reliably from the control, but the trajec-

\footnotetext{
${ }^{1}$ The cut off to define low satisfaction of $0.5 S D$ below the population mean was somewhat arbitrary. We repeated the analyses dividing couples into distressed, defined by the usual convention of scoring $1 S D$ below the population mean on satisfaction, or non-distressed. The pattern of findings was similar as for the analysis with low satisfaction, only the distressed couples showed a reliable increase in satisfaction.
} 
Table 1

Demographics by Condition and High/Low Initial Satisfaction

\begin{tabular}{|c|c|c|c|c|c|c|}
\hline \multirow[b]{2}{*}{ Initial satisfaction condition } & \multicolumn{3}{|c|}{ Low } & \multicolumn{3}{|c|}{ High } \\
\hline & $\begin{array}{c}\text { Control } \\
(n=24)\end{array}$ & $\begin{array}{l}\text { RELATE } \\
(n=21)\end{array}$ & $\begin{array}{l}\text { CoupleCARE } \\
\quad(n=28)\end{array}$ & $\begin{array}{c}\text { Control } \\
(n=34)\end{array}$ & $\begin{array}{l}\text { RELATE } \\
(n=40)\end{array}$ & $\begin{array}{c}\text { CoupleCARE } \\
(n=34)\end{array}$ \\
\hline \multicolumn{7}{|l|}{ Continuous variables, $M(S D)$} \\
\hline Male age & $48.0(11.6)$ & $43.1(12.0)$ & $47.3(10.8)$ & $43.5(11.5)$ & $44.3(11.1)$ & $42.2(10.8)$ \\
\hline Female age & $43.1(10.4)$ & $40.8(10.2)$ & $46.1(11.2)$ & $39.7(11.0)$ & $39.8(9.5)$ & $39.3(10.0)$ \\
\hline Household income $\$ 000$ & $122.5(63.0)$ & $126.2(49.9)$ & $146.2(48.7)$ & $176.1(69.0)$ & $146.4(64.3)$ & $151.5(62.5)$ \\
\hline \multicolumn{7}{|l|}{ Categorical variables, $n(\%)$} \\
\hline Married & $14(58 \%)$ & $18(86 \%)$ & $21(75 \%)$ & $22(65 \%)$ & $28(70 \%)$ & $21(62 \%)$ \\
\hline Male 2nd marriage & $7(29 \%)$ & $4(19 \%)$ & $8(29 \%)$ & $12(35 \%)$ & $12(30 \%)$ & $10(29 \%)$ \\
\hline Female 2nd marriage & $8(33 \%)$ & $4(19 \%)$ & $4(14 \%)$ & $9(26 \%)$ & $6(15 \%)$ & $3(9 \%)$ \\
\hline Male Australian born & $22(92 \%)$ & $17(81 \%)$ & $23(82 \%)$ & $26(76 \%)$ & $34(85 \%)$ & $26(76 \%)$ \\
\hline Female Australian born & $22(92 \%)$ & $16(75 \%)$ & $22(79 \%)$ & $27(79 \%)$ & $34(85 \%)$ & $27(79 \%)$ \\
\hline Male university degree & $14(58 \%)$ & $14(67 \%)$ & $22(79 \%)$ & $28(82 \%)$ & $33(83 \%)$ & $26(76 \%)$ \\
\hline Female university degree & $21(88 \%)$ & $14(67 \%)$ & $26(93 \%)$ & $29(85 \%)$ & $31(78 \%)$ & $27(79 \%)$ \\
\hline
\end{tabular}

tory of RCC is significantly less positive than for the control, and the estimated trajectory of RCC is essentially no change across the 4 years rather than the gradual increase in the control and RELATE.

Turning to the moderation by initial satisfaction, in couples with initially high satisfaction there is no reliable change in satisfaction from pre-RE to post-RE in the control condition, and no difference between conditions in change. The trajectory of relationship satisfaction in the 4 years after RE is for a medium size increase in satisfaction in the control, $d=0.54,95 \%$ CI [0.29-0.79], and there is no difference in trajectory between conditions. By definition, the low satisfaction couples start with lower satisfaction than the high satisfaction couples. In control couples with low initial satisfaction there is a reliably larger increase in mean satisfaction to control high satisfaction couples, $d=0.46,95 \%$ CI [0.08-0.84]. RELATE does not differ reliably in change from the control. RCC increased satisfaction more than the control, with a medium to large effect, $d=0.62,95 \%$ CI [0.09-1.16]. The total increase for couples in RCC was 4.55 points, a large effect, $d=1.08,95 \%$ CI [0.80-1.36]. The slope of the trajectory of relationship satisfaction in the control condition does not differ reliably between the high and low satisfaction couples in the 4 years after RE. The slope of the trajectory does not differ between the control and RELATE, but there is a trend for the trajectory of RCC relative to control to be less positive in low satisfaction couples than the in the other conditions.
The unconditional growth model of mental health had a fixed effect of time, meaning there was an overall change in mean mental health across time, $\chi^{2}(2)=22.74 p<.001$, and a random effect of time, $\chi^{2}(2)=14.73 p<.001$, reflecting that there was variability in the extent of change between couples. Mean mental health increased from pre-RE to post-RE, which relative to normative data in a large Dutch sample with $S D=0.86$ (Lamers, Westrehof, Bohlmeijer, ten Klooster, \& Keyes, 2011), was a small effect, $d=.22,95 \%$ CI [0.12-0.32]. The trajectory from post-RE to 4 years was close to zero, that is, mean mental health remained relatively stable across that time. As shown in Table 2, there is no reliable change in mental health from pre-RE to post-RE in any of the conditions overall, nor for the initially high or low satisfaction couples. Nor is there evidence of change from post-RE to the 4 year follow-up in any of the conditions, for either the high or low satisfaction couples. There might seem to be an anomaly in finding a small pre-RE to post-RE increase in mental health in the unconditional growth model, and yet finding no change in mental health across time in any of the conditions. However, this reflects that there is reduced power to detect what is a small effect in the final model, which is examining change effects separately by condition and low-satisfaction versus high-satisfaction couples.

Figure 2 presents the MLM modeling estimates of relationship satisfaction and mental health trajectories. The pre-RE to post-RE RCC increase in satisfaction for initially low satisfaction couples appears even larger than suggested by the coefficient of the dif-

Table 2

Raw Means (Standard Deviations) for Relationship Satisfaction and Mental Health at Each Assessment

\begin{tabular}{|c|c|c|c|c|c|c|}
\hline \multirow[b]{2}{*}{ Time } & \multicolumn{3}{|c|}{ Relationship satisfaction } & \multicolumn{3}{|c|}{ Mental health } \\
\hline & Control & RELATE & CoupleCARE & Control & RELATE & CoupleCARE \\
\hline Pre & $22.13(4.95)$ & $22.70(5.00)$ & $22.12(5.14)$ & $3.46(.73)$ & $3.49(.80)$ & $3.52(.84)$ \\
\hline Post & $23.01(4.95)$ & $23.67(4.69)$ & $24.96(3.39)$ & $3.67(.73)$ & $3.62(.83)$ & $3.91(.55)$ \\
\hline 6-month follow-up & $21.97(4.70)$ & $22.92(5.40)$ & $24.21(4.33)$ & $3.68(.84)$ & $3.44(1.02)$ & $3.78(.92)$ \\
\hline 12-month follow-up & $23.21(4.43)$ & $23.07(6.20)$ & $23.40(4.03)$ & $3.53(.87)$ & $3.54(.97)$ & $3.78(.88)$ \\
\hline 18-month follow-up & $22.71(5.41)$ & $23.49(5.21)$ & $23.35(5.39)$ & $3.67(.84)$ & $3.75(.89)$ & $3.80(.85)$ \\
\hline 30-month follow-up & $22.53(5.14)$ & $23.49(5.21)$ & $22.93(5.36)$ & $3.61(.84)$ & $3.62(.87)$ & $3.74(.76)$ \\
\hline 48-month follow-up & $22.99(4.49)$ & $22.68(5.80)$ & $22.99(4.83)$ & $3.57(.76)$ & $3.59(.98)$ & $3.63(.86)$ \\
\hline
\end{tabular}

Note. The descriptive statistics ignore the clustering due to couples and are based on available participants per assessment time. 
Table 3

Multilevel Model Coefficients, Standard Errors (in Parentheses) of Relationship Satisfaction and Mental Health After Relationship Education for High and Low Satisfaction Couples

\begin{tabular}{|c|c|c|c|c|c|}
\hline Component & Condition & Satisfaction & $p$ & Mental health & $p$ \\
\hline \multicolumn{6}{|c|}{ Overall effects across high and low satisfaction couples } \\
\hline \multirow[t]{3}{*}{ Pre-RE } & Control & $22.14(.58)$ & - & $3.456(.081)$ & - \\
\hline & RELATE V Control & $.56(.80)$ & .480 & $.045(.114)$ & .693 \\
\hline & RCC V Control & $.00(.80)$ & .997 & $.057(.114)$ & .614 \\
\hline \multirow[t]{3}{*}{ Change } & Control & $-.28(.40)$ & .483 & $.199(.073)$ & $.007^{*}$ \\
\hline & RELATE V Control & $.35(.55)$ & .522 & $-.116(.100)$ & .248 \\
\hline & RCC V Control & $1.86(.57)$ & $.001^{*}$ & $.095(.103)$ & .356 \\
\hline \multirow[t]{3}{*}{ Trajectory } & Control & $.65(.20)$ & $.001^{*}$ & $-.024(.024)$ & .336 \\
\hline & RELATE V Control & $-.35(.28)$ & .211 & $.026(.036)$ & .454 \\
\hline & RCC V Control & $-.66(.28)$ & $.018^{*}$ & $-.019(.034)$ & .587 \\
\hline \multicolumn{6}{|c|}{ Effects on high satisfaction couples } \\
\hline \multirow[t]{3}{*}{ Pre-RE } & Control & $25.29(.47)$ & - & $3.581(.101)$ & - \\
\hline & RELATE V Control & $.18(.63)$ & .773 & $.059(.138)$ & .668 \\
\hline & RCC V Control & $.41(.61)$ & .539 & $.126(.144)$ & .381 \\
\hline \multirow[t]{3}{*}{ Change } & Control & $-.97(.56)$ & .081 & $.156(.096)$ & .105 \\
\hline & RELATE V Control & $.72(.73)$ & .321 & $-.036(.127)$ & .777 \\
\hline & RCC V Control & $.61(.78)$ & .435 & $.086(.135)$ & .523 \\
\hline \multirow[t]{3}{*}{ Trajectory } & Control & $.57(.26)$ & $.031^{*}$ & $-.034(.031)$ & .284 \\
\hline & RELATE V Control & $-.34(.36)$ & .349 & $.001(.043)$ & .968 \\
\hline & RCC V Control & $-.33(.37)$ & .374 & $-.001(.043)$ & .966 \\
\hline \multicolumn{6}{|c|}{ Difference between high and low satisfaction couples } \\
\hline \multirow[t]{3}{*}{ Pre-RE } & Control & $-7.58(.72)$ & $<.001^{*}$ & $-.299(.157)$ & .057 \\
\hline & RELATE V Control & $-.46(1.03)$ & .652 & $-.101(.224)$ & .653 \\
\hline & RCC V Control & $-.29(1.00)$ & .773 & $-.135(.219)$ & .539 \\
\hline \multirow[t]{3}{*}{ Change } & Control & $1.92(.86)$ & $.025^{*}$ & $.106(.149)$ & .479 \\
\hline & RELATE V Control & $-.91(1.21)$ & .451 & $-.260(.210)$ & .217 \\
\hline & RCC V Control & $2.63(1.20)$ & $.028^{*}$ & $.019(.210)$ & .928 \\
\hline \multirow[t]{3}{*}{ Trajectory } & Control & $.21(.42)$ & .623 & $.025(.050)$ & .612 \\
\hline & RELATE V Control & $-.02(.62)$ & .972 & $.110(.076)$ & .153 \\
\hline & RCC V Control & $-.97(.58)$ & .098 & $-.045(.071)$ & .523 \\
\hline
\end{tabular}

Note. $\quad$ Change $=$ change from prerelationship education (Pre-RE) to postrelationship education; Trajectory $=$ points per year from post to 4 year follow-up; RCC $=$ Relate plus CoupleCARE.

${ }^{*} p<.05$.

ference between RCC and control in low satisfaction couples. This is because a number of other coefficients in the model (e.g., RCC vs. control in low satisfaction couples), although not reliably different from zero, all trend for gains in satisfaction to be larger in RCC. Although there are no statistically reliable differences in trajectory across conditions for either low or high satisfaction couples, the trajectory of the low satisfaction couples appears to decline more for RCC than for the other two conditions. Table 4 presents results of the delta method, which combines the parameters in the model to estimate the difference between conditions at each time point, and the standard error of those estimates, separately for low and high satisfaction couples. As shown in Table 4, and presented also in Figure 2, there were no differences between conditions at any time point for high satisfaction couples. In low satisfaction couples RELATE was not reliably different from the control, but RCC produced reliably higher relationship satisfaction than the control at postassessment and 6-month follow-up, and there was a trend in the same direction at 12 months. There were no reliable differences between $\mathrm{RCC}$ and control thereafter, and if anything the trend at 4 year follow-up is for RCC to have lower satisfaction than the control. Thus, there was a clear intervention effect of RCC with low satisfaction couples but that effect attenuated across the next 12 months.

In Figure 2 the pre-RE to post-RE overall increase in mental health is evident. The similarity across conditions of pre-RE to post-RE change, and the similarity in trajectory across conditions to 4 year follow-up are evident. In summary, RCC produced a selective increase in satisfaction in low satisfaction couples that attenuated across the first year; there was a small increase in mental health from pre-RE to post-RE, with no evidence of differential effects across conditions. ${ }^{2}$

Figure 3 shows the reliable change in satisfaction from pre-RE to post-RE for low and high satisfaction couples across conditions. Among treatment completers, higher rates of reliable change were evident in low satisfaction couples in RCC (71\%) than for control $(21 \%)$ or RELATE $(23 \%), \chi^{2}(2)=9.38 p=.009$, but there was

\footnotetext{
${ }^{2}$ We added gender as a dummy variable to the final model equations predicting relationship satisfaction and mental health. We found no gender moderation of any of the effects, although caution must be used in interpreting these null findings as gender was only added after all the other predictors, and hence the power to detect gender effects was modest.
} 

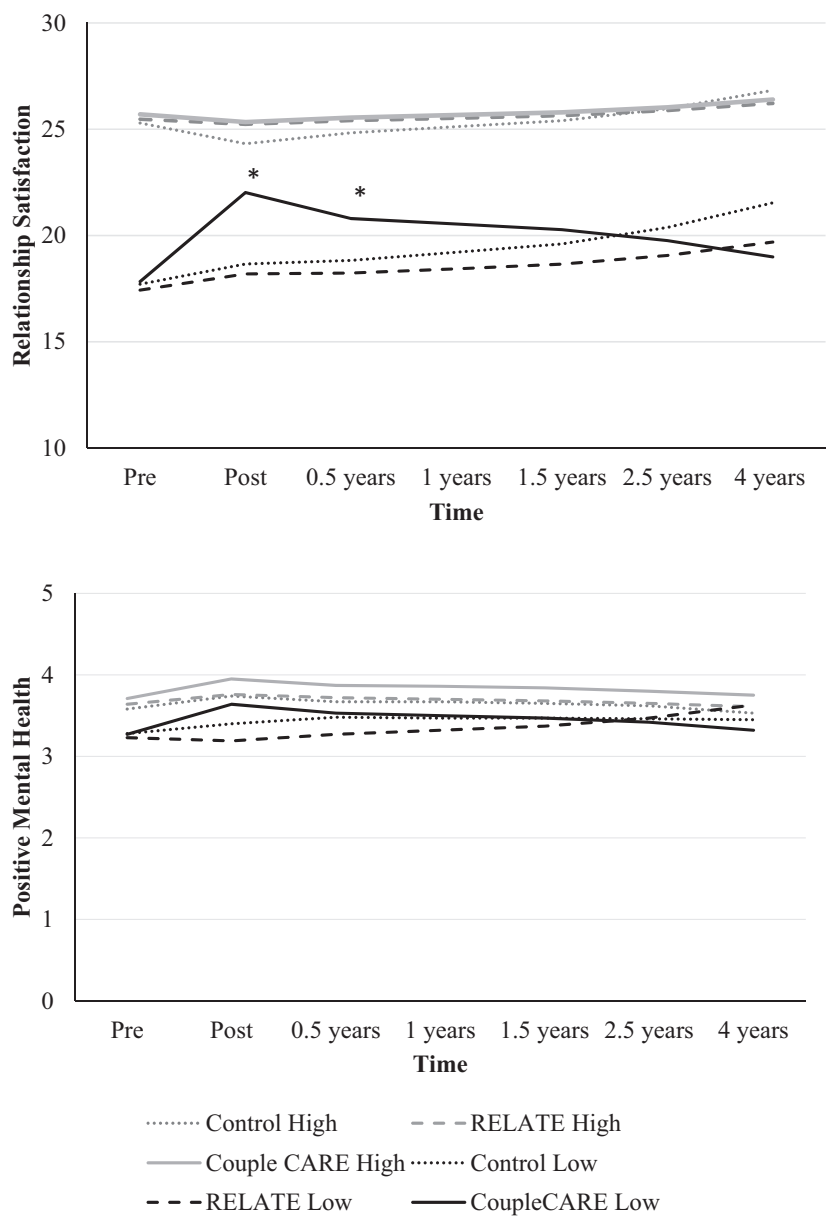

Figure 2. Effect of relationship education on couple satisfaction and mental health across 4 years by initial relationship satisfaction. The only reliable differences between conditions are indicated by ${ }^{*} p<.05$ that RELATE with CoupleCARE (RCC) and control are the same.

no difference between conditions in high satisfaction couples, $\chi^{2}(2)=0.290 p=.865$, and only about $10 \%$ of couples reliably improved.

\section{Discussion}

Contrary to Hypothesis 1, we found no detectable effect of RELATE assessment with feedback on couple relationship satisfaction among couples with low initial satisfaction. There was partial support for Hypothesis 2. As predicted, RCC increased relationship satisfaction in low satisfaction couples, but contrary to prediction the effects of RCC attenuated by the 12-month follow-up to the extent the differences between conditions was no longer statistically significant. Hypothesis 3 was supported, neither RCC or RELATE increased relationship satisfaction in high satisfaction couples. Contrary to Hypothesis 4, neither RELATE nor RCC increased mental health relative to the control condition, and this was true for both low and high relationship satisfaction couples. The effects of RE were not significantly different for women and men across conditions and outcomes.

\section{RE Effects}

RELATE had no effect on relationship satisfaction relative to the guided reading control, which fails to replicate reported effects of assessment with feedback (e.g., Larson et al., 2007; Cordova et al., 2014). Most previous studies had more intensive RE than the RELATE condition in the current study, by including multimodal assessment across multiple sessions (Cordova et al., 2014; Worthington et al., 1995) rather than a single self-report inventory, and some even included brief curriculum-based RE (Cordova et al., 2014). In addition, all previous studies compared assessment with feedback to a wait list control (Larson et al., 2007; Cordova et al., 2014; Worthington et al., 1995), whereas the current study had an active control of guided reading. The combination of a more intensive intervention and not controlling for expectancy effects with a wait list control likely explains the discrepant findings. There is little compelling evidence that assessment with feedback alone produces long-term effects on relationship satisfaction. In contrast, assessment with feedback combined with curriculumbased RE does enhance relationship satisfaction (Busby, Ivey, Harris, \& Ates, 2007; Halford et al., 2010), although it is unclear what contribution the assessment with feedback makes.

The current findings replicate prior findings that CoupleCARE produces an immediate increase in relationship satisfaction (Halford et al., 2010). The selective immediate effect of CoupleCARE with just low satisfaction couples is consistent with other studies in which there were immediate effects of the PREP RE program for initially less satisfied couples (Quirk et al., 2014). Although further replication is needed, it suggests that the observed immediate effect being restricted to low satisfaction couples might be evident across many RE programs.

The current research extends prior findings by being one of only a handful of studies to examine effects of RE for more than 2 years and showed the immediate effects of CoupleCARE attenuated after 12 months. This contrasts with earlier studies showing selective long-term effects of CoupleCARE for high-risk couples at 3.5-year (Petch et al., 2012) and 4-year follow-up (Halford, Sanders, \& Behrens, 2001). The long-term effects in those earlier studies were with couples initially high in satisfaction, who maintained satisfaction at higher levels than the eroding satisfaction evident in control couples, showing a selective long-term effect of preventing erosion of satisfaction. In contrast, in the current study there was limited maintenance of an immediate increase in satisfaction relative to control couples, with the control high satisfaction couples showing increasing satisfaction across time. Attenuation of an immediate increase in satisfaction also was observed by Cordova and colleagues (2014) with their marriage check-up, although in that instance the control couples showed stable satisfaction across time and the RE couples relapsed toward the control couples. A booster session at 12 months re-established enhanced relationship satisfaction, but effects again attenuated through to a 2 year follow-up. Cordova and colleagues (2014) suggest RE is like a marital health check-up, and in the sense that health checks and indicated RE might need to be regular (perhaps annual), their metaphor seems fitting. This is a quite different model of RE delivery than the most common format in which couples attend a single RE course designed to enhance the future trajectory of relationship satisfaction (Halford et al., 2008). The acceptability 
Table 4

Delta Difference Method Comparison of RELATE and CoupleCARE With Control at Each Follow-Up Assessment in Low and High Satisfaction Couples

\begin{tabular}{|c|c|c|c|c|c|c|c|}
\hline \multirow[b]{2}{*}{ Condition } & \multirow[b]{2}{*}{ Time in years } & \multicolumn{3}{|c|}{ Low satisfaction couples } & \multicolumn{3}{|c|}{ High satisfaction couples } \\
\hline & & Difference $(S E)$ & $p$ & $d$ & Difference $(S E)$ & $p$ & $d$ \\
\hline \multirow{5}{*}{ RELATE } & .5 & $-.59(.81)$ & .460 & .14 & $.57(.62)$ & .358 & .14 \\
\hline & 1 & $-.77(.87)$ & .373 & .09 & $.41(.66)$ & .542 & .10 \\
\hline & 1.5 & $-.96(1.00)$ & .338 & .08 & $.24(.75)$ & .752 & .01 \\
\hline & 2.5 & $-1.32(1.36)$ & .334 & .08 & $-.10(.99)$ & .920 & -.02 \\
\hline & 4 & $-1.85(2.02)$ & .360 & .09 & $-.60(1.46)$ & .678 & -.14 \\
\hline \multirow[t]{5}{*}{ CoupleCARE } & .5 & $1.97(.76)$ & $.010^{*}$ & .47 & $.71(.66)$ & .282 & .17 \\
\hline & 1 & $1.32(.81)$ & .104 & .31 & $.55(.70)$ & .434 & .13 \\
\hline & 1.5 & $.67(.92)$ & .462 & .16 & $.39(.79)$ & .623 & .09 \\
\hline & 2.5 & $-.61(1.23)$ & .616 & -.14 & $.06(1.03)$ & .995 & .01 \\
\hline & 4 & $-2.56(1.81)$ & .159 & -.60 & $-.43(1.50)$ & .773 & -.10 \\
\hline
\end{tabular}

Note. Difference $=$ difference between the adjusted group means at each time between the indicated intervention and the control (also referred to as least squares mean in SAS); $S E=$ standard error of the difference; $d=$ effect size of difference.

$* p<.05$

and efficacy of regular brief RE needs to be evaluated, but might well be optimized by a brief flexible delivery program.

In the current study the lack of effects of RE on relationship satisfaction in high satisfaction couples might reflect that RE truly has no benefit for those couples. Alternatively, the null results might reflect a limitation of the relationship satisfaction measure. Item-response theory analyses show that the most widely used relationship satisfaction scales have rapidly declining measurement precision from about 0.5 $S D$ above the population mean (Funk \& Rogge, 2007), which makes detecting positive effects of $\mathrm{RE}$ for high satisfaction couples difficult as much of the variance in couples' scores at the upper ranges is measurement error. ${ }^{3}$ Detecting any effect of RE on trajectory in high satisfaction couples required taking couples who began at a mean of over 25 , which is more than $0.5 S D$ above the population mean, and accelerating the increase observed in the control condition, which would take the means into the range in which measurement precision is particularly poor. As suggested by Fincham and Beach (2010), future research should test whether high functioning relationships can be reliably characterized and assessed. Measures more sensitive to variations in high levels of satisfaction might detect benefits of RE for highly satisfied couples, but clearly no measurable benefits of RE were evident with a standard measure of relationship satisfaction.

Among the low satisfaction couples, the control couples showed a small to medium $(d=.44)$ increase in relationship satisfaction from pre- to post-RE, which was not reliably different to low satisfaction couples who received RELATE. The increases across these two conditions might be regression to the mean. For example, if transient life circumstances led to a dip in satisfaction that prompted couples to present for RE, then those circumstances might change and produce an increase in satisfaction unrelated to RE. Alternatively, the increase in satisfaction might be a nonspecific effect of RE (e.g., couples committing to do something about their relationship increasing satisfaction), or specific effects of the conditions (e.g., reading the book or receiving assessment with feedback changed knowledge to a similar extent).

There was no specific effect of RE on mental health. As noted previously, there is a well-established correlation between mental health and relationship satisfaction (Whisman, 2013), which led us to predict that increased relationship satisfaction might enhance mental health. In the current sample, pre-RE mental health and relationship satisfaction had reliable medium sized correlation for men, $r=.33 p<.01$, and women, $r=.38 p<.01$, which is consistent with prior findings that mental health is associated with relationship satisfaction. Although a few studies have found enhanced mental health after RE (e.g., Braithwaite \& Fincham, 2011), effects typically have been small. Medium to large increases in relationship satisfaction for low satisfaction couples in the current sample did not produce detectable effects on individual mental health.

\section{Limitations of the Research}

There was a difference in the intensity of intervention across the three conditions. The control condition involved reading a relatively brief book and minimal educator contact (just one brief call to prompt reading). The RELATE condition involved reading and discussing the RELATE report with a partner and one session of feedback and goal setting with an educator. RELATE with CoupleCARE involved about $1.5 \mathrm{hr}$ per week of structured activities plus seven review sessions with an educator. The current study had more active controls relative to the waiting list controls used in most RE trials (Halford \& Bodenmann, 2013). However, some nonspecific components of the CoupleCARE program (e.g., the regular couple conversations that are included as part of the program, the ongoing commitment to focus on the relationship, ongoing support by the educator), might produce gains in relationship satisfaction rather than the acquisition of knowledge or skills specific to the program. The assumption that skill-based RE

\footnotetext{
${ }^{3}$ The notion of low measurement precision in upper ranges of a scale is related to, but also is distinct from, a ceiling effect. A ceiling effect refers to when a substantial proportion of the sample have scores approaching the maximum possible score and hence further improvements are not possible (Wang, Zhang, McArdle, \& Salthouse, 2008). With low measurement precision, higher scores might be mathematically possible but the variability between scores at the upper end of the range is primarily measurement error.
} 


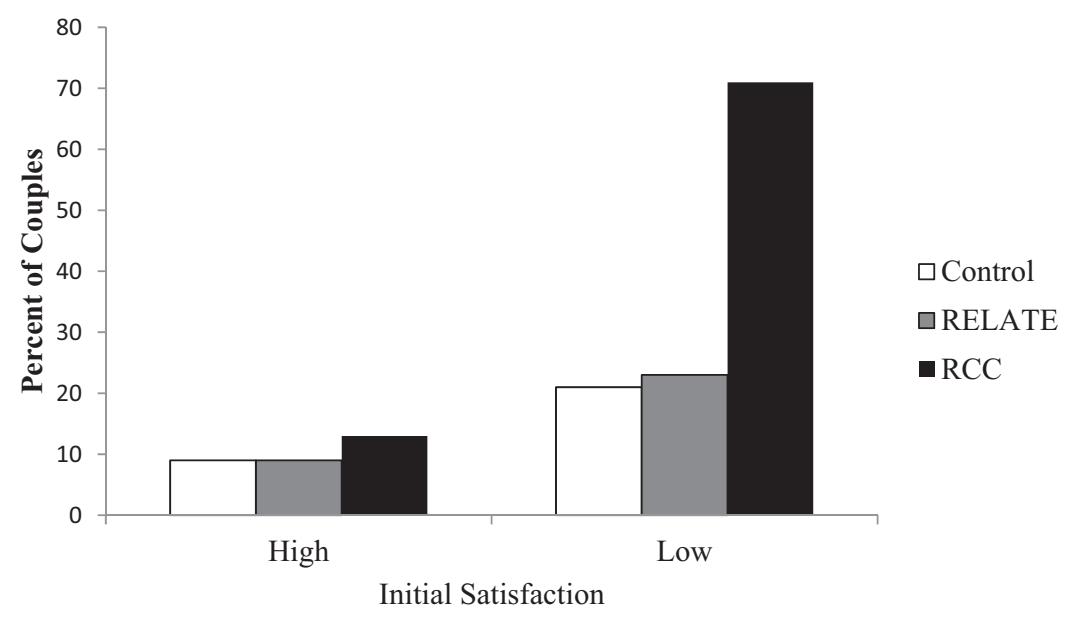

Figure 3. Reliable improvement (change score $>1.96$ standard errors of the change score) from Pre- to Postrelationship Education of Relationship Satisfaction $\times$ Initial Satisfaction and Relationship Education Condition; RCC $=$ Relate with Couple CARE.

teaches skills that have ongoing reinforcement to maintain them, and that these changes enhance the trajectory of future relationship satisfaction, has been challenged by some writers (e.g., Johnson \& Bradbury, 2015). The current pattern of response to RE of a short-term increase in satisfaction that attenuates across time does not fit with the classic social learning assumptions as to why RE has its effects. Future research needs to clarify the mediators of change in RE.

We classified couples as high or low on satisfaction based on the mean of the two partners' satisfaction. Partner satisfaction correlated at $r=.65$, suggesting the mean was a reasonable way to classify couples. However, the system classifies as low satisfaction those couples in which the partners are discrepant, and one partner is high and the other low in satisfaction, as well as couples in which both partners have somewhat low satisfaction. Among the 73 low satisfaction couples, $18(25 \%)$ showed a discrepancy of 1 $S D$ or more on satisfaction at presentation. Lack of power prevents us testing whether the couples' response to RE might vary if both partners have low satisfaction versus if only one partner does, and this possibility should be tested in future research.

The participants were more highly educated than the general population of the country from which they were drawn (Australia), and were predominantly Caucasian. The generalizability of the findings to less educated and culturally diverse couples needs to be assessed in future research. Also, although a substantial proportion of couples had low relationship satisfaction before RE, there were relatively few severely distressed couples. RE usually does not address issues like individual vulnerabilities or interpartner violence, which are problems that often exist in severely distressed couples, and the presence of these problems predicts less improvement after RE (Williamson et al., 2015). Whether RE benefits severely distressed couples requires more research.

\section{Practice Implications}

RE has traditionally been delivered universally, with the aim of helping currently satisfied couples sustain high relationship satisfaction in the long term. However, the current research and other research (Quirk et al., 2014; Williamson et al., 2015) shows the immediate benefits of RE seem to be specific to couples who present with low relationship satisfaction. Given that RE typically is briefer than couple therapy, and the standardized curriculum means it is easier to deliver than couple therapy, RE holds promise as an indicated intervention for mild to moderately distressed couples. However, clearly the loss of intervention effects across time need to be addressed.

The selective and indicated effects of RE, and the absence of compelling evidence of universal effects, suggest the need to target the offering of RE to those most likely to benefit. There have been a number of successful attempts at recruiting high-risk couples, such as couples forming stepfamilies (Lucier-Greer \& AdlerBaeder, 2012), or couples with history of family of origin divorce or violence (Halford et al., 2001). Similarly, the current and other studies (DeMaria, 2005) show couples with low satisfaction can be recruited to participate in RE. Markman and Ritchie (2015) criticize any move to make RE a selective or indicated offering, as this potentially undermines some characteristics of RE (its universality, positive focus) that make it attractive to couples. However, offering RE universally when there is no reliable evidence of its benefits for many currently satisfied couples is hard to justify.

As noted in the introduction, Bradford and colleagues (2015) suggested that, as RE is attracting distressed couples, there is a need for systematic assessment, screening of couples, and additional training of RE providers in clinical skills. As shown in the current and previous studies (Halford et al., 2010) some assessment with feedback can be incorporated into RE effectively, which does allow for discussing with couples their current satisfaction, risk level, and whether undertaking a curriculum-based RE is needed. Providing some training for RE providers on how to have this discussion seems appropriate. In addition, training on how to respond to issues like interpartner violence and partner psychological disorder would be helpful so RE providers can recommend alternative services for couples and individual partners when that is indicated. However, including intervention to manage interpartner violence or individual partner psychopathology as a routine 
part of RE would blur the distinction between RE and couple therapy, and runs the risk of making RE attendance less attractive to couples.

\section{Conclusion}

Results from the current study failed to find any evidence of benefits of RE for high satisfaction couples, and universal offering of RE is not justified by current evidence. Flexible delivery RE had immediate benefits for couples with low initial satisfaction, but those effects attenuated across a 6- to12-month period. Future research needs to test whether booster interventions are acceptable to couples, and whether they are effective in enhancing the longterm maintenance of couple relationship satisfaction by RE as an indicated intervention.

\section{References}

Australian Bureau of Statistics. (2011). Social Indicators. Canberra, Australia: Australian Government Publisher.

Bradford, A. B., Hawkins, A. J., \& Acker, J. (2015). If we build it, they will come: Exploring policy and practice implications of public support for couple and relationship education for lower income and relationally distressed couples. Family Process, 54, 639-654. http://dx.doi.org/10 $.1111 /$ famp. 12151

Braithwaite, S. R., \& Fincham, F. D. (2011). Computer-based dissemination: A randomized clinical trial of ePREP using the actor partner interdependence model. Behaviour Research and Therapy, 49, 126-131. http://dx.doi.org/10.1016/j.brat.2010.11.002

Braukhaus, C., Hahlweg, K., Kroeger, C., Groth, T., \& Fehm-Wolfsdorf, G. (2003). The effects of adding booster sessions to a prevention training program for committed couples. Behavioural and Cognitive Psychotherapy, 31, 325-336. http://dx.doi.org/10.1017/S1352465803003072

Busby, D. M., Holman, T. B., \& Taniguchi, N. (2001). RELATE: Relationship evaluation of the individual, family, cultural, and couple contexts. Family Relations: An Interdisciplinary Journal of Applied Family Studies, 50, 308-316. http://dx.doi.org/10.1111/j.1741-3729.2001 .00308.x

Busby, D. M., Ivey, D. C., Harris, S. M., \& Ates, C. (2007). Self-directed, therapist-directed, and assessment-based interventions for premarital couples. Family Relations: An Interdisciplinary Journal of Applied Family Studies, 56, 279-290. http://dx.doi.org/10.1111/j.1741-3729 .2007.00459.x

Cordova, J. V., Fleming, C. J., Morrill, M. I., Hawrilenko, M., Sollenberger, J. W., Harp, A. G., . . . Wachs, K. (2014). The Marriage Checkup: A randomized controlled trial of annual relationship health checkups. Journal of Consulting and Clinical Psychology, 82, 592-604. http://dx.doi.org/10.1037/a0037097

Cordova, J. V., Scott, R. L., Dorian, M., Mirgain, S., Yaeger, D., \& Groot, A. (2005). The marriage check-up: An indicated preventive intervention for treatment-avoidant couples at risk for marital deterioration. Behavior Therapy, 36, 301-309. http://dx.doi.org/10.1016/S0005-7894(05) 80112-1

DeMaria, R. M. (2005). Distressed couples and marriage education. Family Relations: An Interdisciplinary Journal of Applied Family Studies, 54, 242-253. http://dx.doi.org/10.1111/j.0197-6664.2005.00019.x

Doss, B. D., Rhoades, G. K., Stanley, S. M., \& Markman, H. J. (2009b). Marital therapy, retreats, and books: The who, what, when, and why of relationship help-seeking. Journal of Marital and Family Therapy, 35, 18-29. http://dx.doi.org/10.1111/j.1752-0606.2008.00093.x

Doss, B. D., Rhoades, G. K., Stanley, S. M., Markman, H. J., \& Johnson, C. A. (2009a). Differential use of premarital education in first and second marriages. Journal of Family Psychology, 23, 268-273. http:// dx.doi.org/10.1037/a0014356
Duncan, S. F., Childs, G. R., \& Larson, J. H. (2010). Perceived helpfulness of four different types of marriage preparation interventions. Family Relations: An Interdisciplinary Journal of Applied Family Studies, 59, 623-636. http://dx.doi.org/10.1111/j.1741-3729.2010.00628.x

Fincham, F. D., \& Beach, S. R. H. (2010). Of memes and marriage: Toward a positive relationship science. Journal of Family Theory \& Review, 2, 4-24. http://dx.doi.org/10.1111/j.1756-2589.2010.00033.x

Funk, J. L., \& Rogge, R. D. (2007). Testing the ruler with item response theory: Increasing precision of measurement for relationship satisfaction with the Couples Satisfaction Index. Journal of Family Psychology, 21, 572-583. http://dx.doi.org/10.1037/0893-3200.21.4.572

Georgia, E. J., \& Doss, B. D. (2013). Web-based couple interventions: Do they have a future? Journal of Couple \& Relationship Therapy, 12, 168-185. http://dx.doi.org/10.1080/15332691.2013.779101

Halford, W. K. (2011). Marriage and relationship education: What works and how to provide it. New York, NY: Guilford Press.

Halford, W. K. (2016). CoupleCARE: Couple Commitment and Relationship Enhancement (2nd ed.). Brisbane, Australia: Australian Academic Press.

Halford, W. K., \& Bodenmann, G. (2013). Effects of relationship education on maintenance of couple relationship satisfaction. Clinical Psychology Review, 33, 512-525. http://dx.doi.org/10.1016/j.cpr.2013.02.001

Halford, W. K., Markman, H. J., \& Stanley, S. (2008). Strengthening couples' relationships with education: Social policy and public health perspectives. Journal of Family Psychology, 22, 497-505. http://dx.doi .org/10.1037/a0012789

Halford, W. K., O’Donnell, C., Lizzio, A., \& Wilson, K. L. (2006). Do couples at high risk of relationship problems attend premarriage education? Journal of Family Psychology, 20, 160-163. http://dx.doi.org/10 $.1037 / 0893-3200.20 .1 .160$

Halford, W. K., Pepping, C. A., Hilpert, P., Bodenmann, G., Wilson, K. L., Busby, D., . . Holman, T. (2015). Immediate effects of couple relationship education on low-satisfaction couples: A randomized clinical trial plus an uncontrolled trial replication. Behavior Therapy, 46, 409421. http://dx.doi.org/10.1016/j.beth.2015.02.001

Halford, W. K., Sanders, M. R., \& Behrens, B. C. (2001). Can skills training prevent relationship problems in at-risk couples? Four-year effects of a behavioral relationship education program. Journal of Family Psychology, 15, 750-768. http://dx.doi.org/10.1037/0893-3200.15.4 .750

Halford, W. K., Wilson, K., Watson, B., Verner, T., Larson, J., Busby, D., \& Holman, T. (2010). Couple relationship education at home: Does skill training enhance relationship assessment and feedback? Journal of Family Psychology, 24, 188-196. http://dx.doi.org/10.1037/a0018786

Hawkins, A. J., Blanchard, V. L., Baldwin, S. A., \& Fawcett, E. B. (2008). Does marriage and relationship education work? A meta-analytic study. Journal of Consulting and Clinical Psychology, 76, 723-734. http://dx .doi.org/10.1037/a0012584

Hawkins, A. J., \& Erickson, S. E. (2015). Is couple and relationship education effective for lower income participants? A meta-analytic study. Journal of Family Psychology, 29, 59-68. http://dx.doi.org/10 .1037/fam0000045

Johnson, C. A., Stanley, S. M., Glenn, N. D., Amato, P. A., Nock, S. L., Markman, H. J., \& Dion, M. (Eds.). (2002). Marriage in Oklahoma: 2001 Baseline Statewide Survey on Marriage and Divorce. Stillwater, OK: Bureau for Social Research.

Johnson, M. D. (2012). Healthy marriage initiatives: On the need for empiricism in policy implementation. American Psychologist, 67, 296 308. http://dx.doi.org/10.1037/a0027743

Johnson, M. D., \& Bradbury, T. N. (2015). Contributions of social learning theory to the promotion of health relationships: Asset or liability? Journal of Family Theory \& Review, 7, 13-27. http://dx.doi.org/10 $.1111 /$ jftr. 12057 
Kaiser, A., Hahlweg, K., Fehm-Wolfsdorf, G., \& Groth, T. (1998). The efficacy of a compact psychoeducational group training program for married couples. Journal of Consulting and Clinical Psychology, 66, 753-760. http://dx.doi.org/10.1037/0022-006X.66.5.753

Keyes, C. L., Wissing, M., Potgieter, J. P., Temane, M., Kruger, A., \& van Rooy, S. (2008). Evaluation of the mental health continuum-short form (MHC-SF) in Setswana-speaking South Africans. Clinical Psychology \& Psychotherapy, 15, 181-192. http://dx.doi.org/10.1002/cpp.572

Knutson, L., \& Olson, D. H. (2003). Effectiveness of PREPARE Program with premarital couples in a community setting. Marriage \& Family: A Christian Journal, 6, 529-546.

Lamers, S. M., Westerhof, G. J., Bohlmeijer, E. T., ten Klooster, P. M., \& Keyes, C. L. M. (2011). Evaluating the psychometric properties of the Mental Health Continuum-Short Form (MHC-SF). Journal of Clinical Psychology, 67, 99-110. http://dx.doi.org/10.1002/jclp.20741

Larson, J. H. (2003). The Great Marriage Tune-up Book. San Francisco, CA: Jossey-Bass.

Larson, J. H., Vatter, R. S., Galbraith, R. C., Holman, T. B., \& Stahmann, R. F. (2007). The RELATionship Evaluation (RELATE) with therapistassisted interpretation: Short-term effects on premarital relationships. Journal of Marital and Family Therapy, 33, 364-374. http://dx.doi.org/ 10.1111/j.1752-0606.2007.00036.x

Lucier-Greer, M., \& Adler-Baeder, F. (2012). Does couple and relationship education work for individuals in stepfamilies? A meta-analytic study. Family Relations: An Interdisciplinary Journal of Applied Family Studies, 61, 756-769. http://dx.doi.org/10.1111/j.1741-3729.2012.00728.x

Lustria, M. L. A., Noar, S. M., Cortese, J., Van Stee, S. K., Glueckauf, R. L., \& Lee, J. (2013). A meta-analysis of web-delivered tailored health behavior change interventions. Journal of Health Communication, 18, 1039-1069. http://dx.doi.org/10.1080/10810730.2013.768727

Markman, H. J., \& Ritchie, L. L. (2015). Couple relationship education and couples therapy: Healthy marriage or strange bedfellows? Family Process, 54, 655-671. http://dx.doi.org/10.1111/famp.12191

Mondor, J., Sabourin, S., Wright, J., Poitras-Wright, H., McDuff, P., \& Lussier, Y. (2013). Early termination from couple therapy in a naturalistic setting: The role of therapeutic mandates and romantic attachment. Contemporary Family Therapy: An International Journal, 35, 59-73. http://dx.doi.org/10.1007/s10591-012-9229-z

Morrill, M. I., Eubanks-Fleming, C., Harp, A. G., Sollenberger, J. W., Darling, E. V., \& Cördova, J. V. (2011). The marriage checkup: Increasing access to marital health care. Family Process, 50, 471-485. http:// dx.doi.org/10.1111/j.1545-5300.2011.01372.x

Owen, J., Duncan, B., Anker, M., \& Sparks, J. (2012). Initial relationship goal and couple therapy outcomes at post and six-month follow-up. Journal of Family Psychology, 26, 179-186. http://dx.doi.org/10.1037/ a0026998

Petch, J. F., Halford, W. K., Creedy, D. K., \& Gamble, J. (2012). A randomized controlled trial of a couple relationship and coparenting program (Couple CARE for Parents) for high- and low-risk new parents. Journal of Consulting and Clinical Psychology, 80, 662-673. http://dx .doi.org/10.1037/a0028781

Quirk, K., Strokoff, J., Owen, J. J., France, T., \& Bergen, C. (2014). Relationship education in community settings: Effectiveness with dis- tressed and non-distressed low-income racial minority couples. Journal of Marital and Family Therapy, 40, 442-453. http://dx.doi.org/10.1111/ jmft. 12080

Rabe-Hesketh, S., \& Skrondal, A. (2012). Multilevel and longitudinal modeling using Stata (3rd ed.). College Station, TX: Stata Press.

Rogge, R. D., Cobb, R. J., Lawrence, E., Johnson, M. D., \& Bradbury, T. N. (2013). Is skills training necessary for the primary prevention of marital distress and dissolution? A 3-year experimental study of three interventions. Journal of Consulting and Clinical Psychology, 81, 949961. http://dx.doi.org/10.1037/a0034209

Schulz, M. S., Cowan, C. P., \& Cowan, P. A. (2006). Promoting healthy beginnings: A randomized controlled trial of a preventive intervention to preserve marital quality during the transition to parenthood. Journal of Consulting and Clinical Psychology, 74, 20-31. http://dx.doi.org/10 .1037/0022-006X.74.1.20

Snyder, D. K., \& Halford, W. K. (2012). Evidence-based couple therapy: Current status and future directions. Journal of Family Therapy, 34, 229-249. http://dx.doi.org/10.1111/j.1467-6427.2012.00599.x

Stanley, S. M., Amato, P. R., Johnson, C. A., \& Markman, H. J. (2006) Premarital education, marital quality, and marital stability: Findings from a large, random household survey. Journal of Family Psychology, 20, 117-126. http://dx.doi.org/10.1037/0893-3200.20.1.117

Trillingsgaard, T., Baucom, K. J. W., Heyman, R. E., \& Elklit, A. (2012), Relationship interventions during the transition to parenthood: Issues of timing and efficacy. Family Relations: An Interdisciplinary Journal of Applied Family Studies, 61, 770-783. http://dx.doi.org/10.1111/j.17413729.2012.00730.x

Wang, L., Zhang, Z., McArdle, J. J., \& Salthouse, T. A. (2008). Investigating ceiling effects in longitudinal data analysis. Multivariate Behavioral Research, 43, 476-496. http://dx.doi.org/10.1080/ 00273170802285941

Whisman, M. (2013). Relationship discord and the prevalence, incidence, and treatment of psychopathology. Journal of Social and Personal Relationships, 30, 163-170. http://dx.doi.org/10.1177/026540751 2455269

Whisman, M. A., Beach, S. R. H., \& Snyder, D. K. (2008). Is marital discord taxonic and can taxonic status be assessed reliably? Results from a national, representative sample of married couples. Journal of Consulting and Clinical Psychology, 76, 745-755. http://dx.doi.org/10.1037/ 0022-006X.76.5.745

Williamson, H. C., Rogge, R. D., Cobb, R. J., Johnson, M. D., Lawrence, E., \& Bradbury, T. N. (2015). Risk moderates the outcome of relationship education: A randomized controlled trial. Journal of Consulting and Clinical Psychology, 83, 617-629. http://dx.doi.org/10.1037/a0038621

Worthington, E. L., McCullough, M. E., Shortz, J. L., Mindes, E. J., Sandage, S. J., \& Chartrand, J. M. (1995). Can couples assessment and feedback improve relationships? Assessment as a brief relationship enrichment procedure. Journal of Counseling Psychology, 42, 466-475. http://dx.doi.org/10.1037/0022-0167.42.4.466

Received March 11, 2016

Revision received December 6, 2016

Accepted December 8, 2016 